\title{
Recent genetic advances in early-onset dystonia
}

\section{Dora Steel MRCPCH, ${ }^{1,2}$ Manju A Kurian PhD ${ }^{1,2}$}

1. Developmental Neurosciences, UCL Great Ormond Street Institute of Child Health, London, UK.

2. Clinical Neurosciences, Great Ormond Street Hospital for Children, London, UK.

\section{Corresponding author:}

Prof Manju Kurian

NIHR Research Professor and UCL Professor of Neurogenetics

Honorary Consultant Paediatric Neurologist

UCL Great Ormond Street Institute of Child Health

30 Guilford Street

London

United Kingdom

WC1N 1EH

manju.kurian@ucl.ac.uk

Tel: 00442079052876

Word count: 3363

Funding: No specific funding was received for this work. MAK and DS are both funded through an NIHR professorship and MAK's research group also benefits from funding from the Sir Jules Thorn Trust and Rosetrees Trust. 


\section{Introduction}

Dystonia has been defined as "sustained or intermittent muscle contractions causing abnormal, often repetitive, movements, postures, or both" ${ }^{1}$ It is considered a symptom rather than a diagnosis and underlying causes range from psychological disorders through iatrogenesis and acquired brain injury to a wide range of genetic and metabolic conditions. Dystonic conditions are now stratified by the Albanese classification system along two main axes: aetiology, and clinical characteristics. ${ }^{1}$ Among the latter, two key distinctions are made between (i) dystonia which occurs with or without other neurological or systemic features, and (ii) dystonia which is "isolated" - that is, the only movement disorder present - or "combined" - that is, occurring together with other movement disorders such as spasticity or parkinsonism.

In clinical terms, early-onset genetic dystonia embraces both isolated and combined forms of dystonia, as well as those with and without associated features. Indeed, for dystonic conditions of childhood onset, Albanese et al's distinctions between isolated and more complex forms of dystonia are increasingly hard to sustain. With the possible exception of THAP1 (DYT-6), virtually all forms of early-onset genetic dystonia have some association with neurodevelopmental, psychiatric or physical comorbidity, and commonly manifest with more than one type of movement disorder in childhood. There is thus a continuum between disorders where dystonia is the key feature, with minimal comorbidities, through multisystem diseases where dystonia is one symptom of several, to disorders where dystonia can occur but is not usually the main feature. The DYT- numbering system for dystonic disorders does not accurately reflect this complexity, and increasingly the use of gene-names, together with a formulation of the clinical issues, is preferred.

It is just over thirty years since the first specific genetic locus responsible for a "primary" or more-or-less isolated - dystonia was identified. ${ }^{2,3}$ In the time since "the DYT-1 gene", now known to be TOR1A, was discovered through painstaking linkage analysis in affected families, our understanding of genetic causes of dystonia has changed beyond recognition. The advent of next-generation sequencing (NGS) has hugely expanded access to detailed genetic testing, and new genes - or more correctly, new gene-disease relationships - are 
discovered every year. Genetic tests are now widely considered a key part of the investigation of unexplained dystonia.

Nevertheless, a high proportion of cases of dystonia believed to be genetic in origin remain unsolved. In a recent published cohort, investigated with whole-genome sequencing (WGS), a genetic diagnosis was found for just $11.7 \%$ of patients. ${ }^{4}$ Clearly, therefore, many gaps remain in our understanding of the genetics of dystonia. Whether these gaps primarily reflect undiscovered Mendelian disorders, or other complexities of aetiology, remains a moot point.

This review will focus on advances within the NGS era, and in particular, gene discoveries within the last two years. These include the description of several new genetic disorders which can cause early-onset dystonia, but also improvements in our understanding of some known genetic disorders in which dystonia has previously been an unrecognised or underrecognised feature. We will briefly discuss the implications of the changing genetic landscape for clinicians and patients, as well as possible reasons why rates of diagnosis remain relatively low.

\section{Advances in gene discovery in the next generation sequencing era}

The increasing accessibility of NGS techniques, especially WGS and whole-exome sequencing (WES) over the last 10 years, has led to an acceleration in identification of new disease genes. The causative genes for DYT-2,-4, -10 and -24-28 were all identified since 2010, ${ }^{5-13}$ all using NGS techniques (Table 1). In some cases, this has facilitated the identification of specific treatments which are effective for particular genetically defined disorders: examples include the use of carbamazepine for PRRT2-related episodic kinesigenic dyskinesia, ${ }^{14}$ and deep-brain stimulation for KMT2B-related dystonia. ${ }^{13}$ It is notable, however, that the latest disorder to be assigned a DYT- number - DYT-28, caused by mutations in $K M T 2 B^{12,13}$ - was described in 2016 , suggesting progress in gene discovery may have begun to falter.

\section{Novel gene discovery}


At least eight new genetic disorders in which dystonia may be a feature have been described in the last two years (Table 2). All of these are complex conditions of which dystonia is one symptom among several: significant neurodevelopmental impairment is a feature of all eight, and epilepsy of five. It should be noted that two of these conditions (those caused by mutations in PI4K2A and CAMK4) have so far been reported in only a single family, and the gene-disease relationship cannot therefore yet be regarded as firmly established.

\section{GSX2}

Agenesis of the basal ganglia is a rare, severe cerebral malformation presenting with dystonic tetraparesis, the aetiology of which has until now been obscure. De Mori et al ${ }^{15}$ reported two unrelated patients agenesis of the globi pallidi and putamina, with different homozygous mutations - one missense, one nonsense - in GSX2, a homeobox transcription factor. Both children had severe progressive generalised dystonia from early infancy, together with profound neurodevelopmental impairment: neither acquired any speech, or sat independently.

\section{IRF2BPL}

Marcogliese et al $^{16}$ report seven unrelated patients with heterozygous variants in IRG2BPL, which encodes a gene required for ubiquitination, the process by which proteins are tagged for degradation by the proteasome. Five individuals had protein-truncating variants and all of these displayed significant motor and cognitive regression between two and 10 years of age, accompanied by epilepsy and a progressive movement disorder which variably included dystonia, ataxia, spasticity and choreoathetosis. Two other individuals had missense variants and a slightly milder course with slower progression. MRI scans showed gradually progressive loss of white matter volume, but without the changes in signal that would suggest a leukodystrophy. In a fly model, knockdown of the equivalent gene led to progressive abnormalities of motor behaviour.

\section{ZNF142}

Seven patients from four families were found to have biallelic mutations in this zinc-finger transcription factor. ${ }^{17}$ All had moderate-to-severe intellectual disability and five of the seven 
developed epilepsy. Three children (from two families) developed dystonia at between one and four years of age, but in other respects the disorder did not appear to be progressive, and there were no syndromic features.

\section{DEGS1}

19 patients from 13 families presented within the first two years of life with a hypomyelinating leukodystrophy. ${ }^{18}$ Epilepsy occurred in most of the children and all had significant psychomotor delay. Several patients died within the first decade of life. 12 of the patients developed dystonia, while 18 had spasticity. All were found to have biallelic mutations affecting DEGS1, which encodes an enzyme required for synthesis of ceramides from sphingolipids. Several other errors of sphingolipid metabolism are implicated in neurological diseases, including Niemann-Pick disease, Krabbe's and metachromatic leukodystrophy (in each of which dystonia may be a feature).

\section{VPS13D}

Biallelic VPS13D mutations were identified in 12 patients from 7 families who presented with gait disturbance characterised by spasticity and ataxia. ${ }^{19}$ Axonal neuropathy was present in several and two had intellectual disability. Saccadic intrusions were a prominent feature. One patient within this group developed cervical dystonia and a further dystonic patient has since been described..$^{20}$ VPS13D plays a role in autophagy and specifically mitochondrial clearance. ${ }^{21}$

\section{VAMP2}

De novo heterozygous mutations in VAMP2, which encodes a SNARE protein involved in release of synaptic vesicles, were reported in five unrelated individuals. ${ }^{22}$ All had moderate to severe intellectual disability, early-onset hypotonia and stereotypies and $3 / 5$ had epilepsy. Three had hyperkinetic movement disorders: the main feature was chorea but one also exhibited dystonic posturing.

\section{PI4K2A}

Two siblings from a single consanguineous family presented in infancy with subtle dysmorphic features, severe developmental delay, irritability, facial dyskinesia and 
intermittent dystonia. Both went on to develop myoclonus and epilepsy. They shared a homozygous nonsense variant in $P 14 K 2 A$, a gene involved in membrane trafficking and neuronal survival. ${ }^{23}$ Although the pathogenicity of this variant is plausible, it cannot be considered proven in the absence of either a second affected family or extensive functional analysis.

\section{CAMK4}

A single patient with an unexplained dystonic/dyskinetic movement disorder and intellectual disability was found to have a de novo splice site variant in CAMK4. ${ }^{24}$ CAMK4 is a calcium/calmodulin dependent protein kinase highly expressed in brain and mediates intracellular calcium signalling: the mutation was predicted to lead to gain of function. Again, this should be considered a plausible but unproven candidate gene for dystonia.

\section{Recognition of dystonia within the phenotypes of other conditions}

Just as important as the description of new genetic disorders is the identification of new or variant phenotypes within established ones. There are several instances where dysfunction of a particular gene has initially been linked to one condition - for example, epilepsy, or neurodevelopmental delay - but has subsequently been found also be relevant to other manifestations, including dystonia. In some cases this reflects the discovery of a wholly new a distinct second disease phenotype for a gene, but in most it is due to our improved knowledge of a continuous phenotypic spectrum.

\section{NUBPL}

Many mitochondrial disorders are well-established causes of dystonia, ${ }^{25}$ but others have been recognised only very recently. For example, biallelic variants in NUBPL have been known to cause complex I deficiency for a decade, ${ }^{26}$ but the specific presentation of bilateral striatal necrosis and severe progressive dystonia was described only in $2019 .^{27}$

\section{ATP8A2}

Biallelic mutations in ATP8A2, an ATPase required for translocation of phospholipids across membranes, were reported in cerebellar atrophy, mental retardation and disequilibrium 
syndrome in a single family in $2013 .{ }^{28}$ Dystonia did not form part of the original description but in a more recent case series ${ }^{29}$ about a quarter of patients were dystonic, and nearly all had some form of hyperkinetic disorder, most commonly chorea. Other features included hypotonia, severe intellectual disability and progressive optic atrophy.

\section{KIF1A}

KIF1A mutations have been described in a number of presentations including severe infantonset neurodegenerative disorders ${ }^{30}$ and hereditary spastic paraparesis. ${ }^{31}$ Dystonia may occur at both the milder and less severe ends of this spectrum but has only recently been described in any detail. ${ }^{32}$

\section{GRIN2A}

Heterozygous variants are known to cause a range of epilepsy syndromes, most distinctively Landau-Kleffner syndrome. More recently, a novel missense variant in GRIN2A was identified in two children who had dystonia and moderate global developmental delay but no history of epilepsy or EEG abnormalities. ${ }^{33}$

\section{Epilepsy-dyskinesia syndromes}

A number of conditions which were initially recognised as genetic forms of epilepsy are now known to involve movement disorders as well. ${ }^{34}$ In some cases, the movement disorder may be the presenting complaint, or may be more disruptive to the patient and family than the epilepsy itself. Distinguishing between epileptic phenomena and paroxysmal abnormal movements can present a diagnostic challenge but is important as it may radically alter the therapeutic approach.

Examples include RHOBTB2-related disorders: initially, RHOBTB2 variants were reported in early infantile epileptic encephalopathy type $64,{ }^{35}$ but it is becoming clear that for many patients a complex movement disorder, including dystonia, dyskinesia, episodic weakness and paroxysmal movements, is an equally salient feature. ${ }^{36}$ 
TBC1D24-related disorders are known to range from epileptic encephalopathy to isolated hearing loss, ${ }^{37}$ but have also recently been reported in paroxysmal movement disorders, especially exercise-induced dystonia. ${ }^{38}$

Mutations in $K C N T 1$ have been known to cause both malignant migrating partial seizures of infancy and nocturnal frontal lobe epilepsy since 2012,39 but in 2019 it was also reported in a case of life-threatening status dystonicus - without ictal EEG correlate - in a child with epileptic encephalopathy. ${ }^{40}$

\section{Current approach to diagnosis of genetic dystonias}

Faced with a patient with early-onset dystonia, whether in the context of other symptoms or not, the clinician faces a dilemma. Traditional neurological practice favours using clinical assessment to reach a potential diagnosis and then confirming or excluding it through targeted testing. In many settings, this remains an effective and efficient option, but in the field of genetic disorders it is arguably outdated.

As the number of potential diagnoses - many of them so rare that a neurologist cannot expect to encounter more than one case in a career - increases, clinical "spot-diagnosis" becomes more challenging. Meanwhile, speed and accessibility of genetic investigations is improving. Therefore, once a clinician has formulated a clinical diagnosis of "dystonia, likely genetic", broad-spectrum genetic testing is a reasonable next step. Next-generation sequencing has now reached the point where panel-based testing is usually cheaper than testing individual candidate genes, unless only one or two possible diagnoses are suspected.

Having decided on genetic testing, however, the difficulty is not over: the clinician must decide which test is most appropriate. A number of genetic panels for dystonia and other early-onset movement disorders are now available. They provide a time- and cost-effective way to test for several conditions at once, but they still have certain limitations.

Firstly, they require the clinician to formulate the patient's presentation in terms of one main clinical feature. Because dystonia so often occurs in the context of a complex disorder, 
this can be problematic and may lead to false negative results. For example, most currentlyused genetic panels for dystonia do not include genes such as GRIN2A, KIF1A or NUBPL. All three might be found on an epilepsy panel, but if the patient had no history of epilepsy this would naturally not be requested.

Secondly, panels struggle to keep pace with the rate of gene discovery. The numbers of genes included are increasing rapidly - the Sheffield dystonia panel included 28 genes in 2018 , and 73 genes in $2019^{41}$ - but arguably not rapidly enough. There is also a lack of standardisation between commercially available panels: the Centogene dystonia panel includes 88 genes $^{42}$ while the equivalent offered by Invitae has just $23 .{ }^{43}$

Increasingly, as access, cost and speed improve, WGS or WES are becoming first choices for investigation. Here again, though, there are pitfalls. It is important to understand that while the whole genome (or exome) is sequenced, not every gene will be analysed or reported on. Most WES and WGS still rely on "virtual panels" - gene lists closely analogous to those used in panel testing - to guide interrogation of the data. Thus they are subject to some of the same limitations: if a gene is not included in the panel applied, it will be missed.

There are also technical limitations. Certain parts of the genome are hard to sequence accurately (for example, because they are highly repetitive or rich in GC bases), and certain categories of variant, such as triplet repeat expansions and some structural variants, are hard to detect. Changes in gene copy number (i.e. whole-gene deletions and duplications) are also not always reliably detected, although they can be an important mechanism in some genetic dystonias. ${ }^{4}$

Finally, clinicians should keep in mind that genetic investigation should be an iterative process. The fact that no pathogenic variant can be found with the current state of knowledge and technology does not mean that none will be found next year. Arguably, reinterrogation of WGS or WES data (not necessarily re-sequencing) should take place on a regular basis for all unsolved patients, to reflect the fact that new genes and phenotypes will have been identified in the interval. 


\section{The direction of research}

Genetic dystonias are aetiologically diverse, ranging from disorders of neurotransmitter synthesis/release/uptake ${ }^{44}$ and other synaptic abnormalities ${ }^{45}$ to abnormalities affecting chromatin modification, ${ }^{13}$ cytoskeletal function ${ }^{46}$ and mitochondrial metabolism. ${ }^{47}$ This likely reflects the fact that control of voluntary movement is a high-level process which can only function correctly when all the underlying key cellular elements and neural networks are in place. The broad spectrum of biological pathways that may be disrupted in dystonia further complicates the search for novel genes.

Although new forms of genetic dystonia are still being identified, it is clear that many more are currently missed: hence the high rate of unsolved patients. There may be a number of reasons for this, including incomplete penetrance, complex (e.g. digenic, polygenic or mosaic) inheritance, difficulty recognising extremely rare and/or variable phenotypes, and variant types (such as intronic variants) which elude current analysis techniques. These are summarised in Table 3. Ability to create and analyse very large, often population-wide, datasets will overcome some of these obstacles. Gene-hunting will continue, but will also need to adapt and become more sophisticated if it is to identify the full range of genetic causes of disease.

Identification of a gene, of course, is only the first step in understanding the aetiology of a disorder. The chain of causation between a genetic mutation and a complex failure of voluntary motor control systems generally has many links. Here, too, research techniques are moving rapidly: for example, in the last 10 years development of neurons, and even three-dimensional organoids, derived from induced pluripotent stem cells (iPSC) have provided researchers with a new way to model the cellular and molecular mechanisms of disease, and to explore potential therapies in vitro. ${ }^{48}$

\section{Implications for patients and families}

The clinical utility of a diagnosis - even where it cannot direct disease-modifying treatment - is now widely acknowledged. It provides for accurate prognostication, allows genetic counselling, and brings to an end the diagnostic odyssey which, for patients with rare 
disorders, may have lasted many years. As discussed above, it may also allow customised selection of an appropriate therapy from among the available options. Moreover, targeted precision-medicine interventions such as gene therapy are now on the horizon for a range of neurological disorders, and access to these as they become available will depend on accurate diagnosis. Self-evidently, diagnoses can only be made for diseases which have been recognised and described.

A less dramatic development, but also beneficial to patients and families, is the proliferation of single-gene support groups. These are often global and exist primarily on social media, although meetings and activities may take place in the real world as well, and they are a useful source of information and support to many patients and families. Where a patient's primary care physician and even their neurologist may never have encountered anyone else with the same disorder, the pooled expertise of several affected families may be very valuable. Virtually every gene referred to in this article - with the exception of those discovered within the past two years - now has its own dedicated support group, and this trend is likely to increase.

The corollary of this, however, is that undiagnosed patients - or rather, patients who have a clinical diagnosis but not a genetic one - risk being left behind, with less targeted support than their aetiologically-confirmed peers. This is both a motivation to improve rates of diagnosis, and a reason to ensure that health systems recognise patients' care needs and entitlements based on clinical need, rather than a diagnostic label.

\section{Conclusion}

Several new genetic causes of dystonia - all occurring as part of a complex neurological phenotype - have been identified in the last two years, and several more are likely to be discovered in the next two. Discovery of Mendelian gene-phenotype relationships will doubtless continue, but may become more challenging now that many of the lowesthanging fruit have been identified. It also is notable that a list of the most recent discoveries does not include any new forms of isolated dystonia, or dystonia without other systemic or neurological features, even while a majority of such cases remain unsolved. 
In order to reach specific diagnoses for majority of patients with dystonia, new approaches will be required. Bioinformatic techniques are likely to form the basis of this, allowing us to process the increasingly large datasets we can access and detect more complex and nuanced associations. Gene identification remains vitally important as the first step - but only the first step - towards understanding a disease process, and ultimately modifying it to improve patient outcomes.

The idea that a single gene corresponds to a single disease phenotype is increasingly outdated. Increasing breadth and complexity is being discovered within the phenotypic spectra of many genes. It seems reasonable to predict that some of the "missing" causes of dystonia will be found within genes to which other pathological phenotypes have already been assigned.

Neurologists caring for patients with suspected genetic dystonias need to remain au fait with the progress of the field, but it is no longer realistic to aim for clinical diagnosis to the level of predicting the affected gene in all cases. Instead, they should aim to use NGS-based testing to their patients' best advantage, including treating it as an iterative process with reanalysis of unsolved patients at regular intervals. Through these means we can hope to improve the proportion of patients who achieve a prompt and specific diagnosis, and simultaneously move towards enabling precise understanding and precision intervention for their condition.

\section{References}

1. Albanese A, Bhatia K, Bressman B et al. Phemonenology and classification of dystonia: a consensus update. Mov Disord, 2013. 28(7): 863-873.

2. Kramer PL, Ozelius L, Risch N et al. Gene for dystonia in Ashkenazi Jewish population located on chromosome 9q. Am J Hum Genet, 1989. 45: A147.

3. Ozelius LJ, Hewett JW, Page CE et al. The early-onset torsion dystonia gene (DYT1) encodes an ATP-binding protein. Nature Genet, 1997. 17:40-48.

4. Kumar KR, Davis RL, Tchan MC et al. Whole genome sequencing for the genetic diagnosis of heterogenous dystonia phenotypes. Parkinsonism Relat Disord, 2019. 69:111-118.

5. Charlesworth G, Angelova PR, Bartolome-Robledo F et al. Mutations in HPCA cause autosomalrecessive primary isolated dystonia. Am J Hum Genet, 2015. 96: 657-665. 
6. Hersheson J, Mencacci NE, Davis M, et al. Mutations in the autoregulatory domain of beta-tubulin 4a cause hereditary dystonia. Ann Neurol, 2013. 73: 546-553.

7. Chen WJ, Lin Y, Xiong ZQ et al. Exome sequencing identifies truncating mutations in PRRT2 that cause paroxysmal kinesigenic dyskinesia. Nature Genet, 2011. 43: 1252-1255.

8. Charlesworth G, Plagnol V, Holmstrom KM et al. Mutations in ANO3 cause dominant craniocervical dystonia: ion channel implicated in pathogenesis. Am J Hum Genet, 2012. 91:10411050.

9. Fuchs T, Saunders-Pullman R, Masuho I et al. Mutations in GNAL cause primary torsion dystonia. Nature Genet, 2013. 45: 88-92.

10. Mencacci NE, Rubio-Agusti I, Zdebik A et al. A missense mutation in KCTD17 causes autosomal dominant myoclonus-dystonia. Am J Hum Genet, 2015. 96:938-947.

11. Zech M, Lam DD, Francescatto L et al. Recessive mutations in the alpha-3 (VI) collagen gene COL6A3 cause early-onset isolated dystonia. Am J Hum Genet, 2015. 96:883-893.

12. Zech M, Boesch S, Maier EM et al. Haploinsufficiency of KMT2B, Encoding the Lysine-Specific Histone Methyltransferase 2B, Results in Early-Onset Generalized Dystonia. Am J Hum Genet, 2016. 99(6):1377-1387.

13. Meyer E, Carss KJ, Rankin J et al. Mutations in the histone methyltransferase gene KMT2B cause complex early-onset dystonia. Nat Genet, 2017. 49: 223-237.

14. Silveira-Moriyama L, Gardiner AR, Meyer E et al. Clinical Features of Childhood-Onset Paroxysmal Kinesigenic Dyskinesia With PRRT2 Gene Mutations. Dev Med Child Neurol, 2013. 55(4):327-34.

15. De Mori R, Severino M, Mancardi MM et al. Agenesis of the putamen and globus pallidus caused by recessive mutations in the homeobox gene GSX2. Brain, 2019, 142(10):2965-2978.

16. Marcogliese PC, Shashi V, Spillmann RC et al. IRF2BPL Is Associated with Neurological Phenotypes. Am J Hum Genet, 2018. 103(2): 245-260.

17. Khan K, Zech M, Morgan AT et al. Recessive variants in ZNF142 cause a complex neurodevelopmental disorder with intellectual disability, speech impairment, seizures, and dystonia. Genet Med, 2019, 21(11): 2532-2542.

18. Pant DC, Dorboz I, Schluter A, Fourcade S. Loss of the sphingolipid desaturase DEGS1 causes hypomyelinating leukodystrophy. J Clin Invest, 2019. 129(3): 1240-1256

19. Seong $E$, Insolera $R$, Dulovic $M$ et al. Mutations in VPS13D lead to a new recessive ataxia with spasticity and mitochondrial defects. Ann Neurol, 2018. 83(6): 1075-1088.

20. Koh K, Ishiura H, Shimazaki H, et al. VPS13D-related disorders presenting as a pure and complicated form of hereditary spastic paraplegia. Mol Genet Genomic Med, 2020. 8(3):e1108.

21. Anding AL, Wang C, Chang TK et al. Vps13D Encodes a Ubiquitin-Binding Protein that Is Required for the Regulation of Mitochondrial Size and Clearance. Curr Biol, 2018. 28(2):287-295 
22. Salpietro V, Malintan NT, Llano-Rivas I, et al. Mutations in the Neuronal Vesicular SNARE VAMP2 Affect Synaptic Membrane Fusion and Impair Human Neurodevelopment. Am J Hum Genet, 2019. 104(4):721-730.

23. Alkhater RA, Schere SW, Minassian BA, Walker S. PI4K2A deficiency in an intellectual disability, epilepsy, myoclonus, akathisia syndrome. Ann Clin Transl Neurol, 2018. 5(12): 1617-1621.

24. Zech M, Lam DD, Weber S, et al. A unique de novo gain-of-function variant in CAMK4 associated with intellectual disability and hyperkinetic movement disorder. Cold Spring Harb Mol Case Stud, 2018. 4(6): a003293.

25. Tranchant C, Anheim M. Movement disorders in mitochondrial diseases. Rev Neurol (Paris), 2016. 172(8-9):524-529.

26. Calvo SE, Tucker EJ, Compton AG et al. High-throughput, pooled sequencing identifies mutations in NUBPL and FOXRED1 in human complex I deficiency. Nature Genet, 2010. 42: 851-858.

27. Balint B, Charlesworth G, Stamelou M et al. Mitochondrial complex I NUBPL mutations cause combined dystonia with bilateral striatal necrosis and cerebellar atrophy. Eur J Neurol, 2019. 26(9):1240-1243.

28. Onat OE, Gulsuner S, Bilguvar K et al. Missense mutation in the ATPase, aminophospholipid transporter protein ATP8A2 is associated with cerebellar atrophy and quadrupedal locomotion. Europ J Hum Genet, 2013. 21:281-285.

29. McMillan HJ, Telegrafi A, Singleton A et al. Recessive mutations in ATP8A2 cause severe hypotonia, cognitive impairment, hyperkinetic movement disorders and progressive optic atrophy. Orphanet J Rare Dis, 2018. 13:86.

30. Okamoto N, Miya F, Tsunoda et al. KIF1A mutation in a patient with progressive neurodegeneration. J Hum Genet, 2014. 59(11):639-41.

31. Citterio A, Arnoldi A, Panzeri E et al. Variants in KIF1A gene in dominant and sporadic forms of hereditary spastic paraparesis. J Neurol, 2015. 262(12):2684-90.

32. Nemani T, Steel D, Kaliakatsos M et al. KIF1A-related disorders in children: a wide spectrum of central and peripheral nervous system involvement. J Peripher Nerv Syst, 2020. Epub ahead of print.

33. Fernández-Marmiesse $A$, Kusumoto $H$, Rekarte $S$ et al. A novel missense mutation in GRIN2A causes a non-epileptic neurodevelopmental disorder. Mov Disord, 201833(6): 992-999.

34. Papandreou A, Danti FR, Spaull R, et al. The expanding spectrum of movement disorders in genetic epilepsies. Dev Med Child Neurol, 2020. 62(2):178-191.

35. Straub J, Konrad EDH, Gruner J et al. Missense variants in RHOBTB2 cause a developmental and epileptic encephalopathy in humans, and altered levels cause neurological defects in Drosophila. Am J Hum Genet, 2018. 102:44-57.

36. Zagaglia S, Steel D, Krithika S et al. RHOBTB2 mutations cause movement disorders that mimic alternating hemiplegia of childhood. Unpublished manuscript in preparation. 
37. Rehman AU, Santos-Cortez RL, Morell RJ et al. Mutations in TBC1D24, a gene associated with epilepsy, also cause nonsyndromic deafness DFNB86. Am J Hum Genet, 2014.94(1):144-52.

38. Lüthy K, Mei D, Fischer B et al. TBC1D24-TLDc-related epilepsy exercise-induced dystonia: rescue by antioxidants in a disease model. Brain, 2019. 142(8):2319-2335.

39. Gertler T, Bearden D, Bhattacharjee A, Carvill G. KCNT1-Related Epilepsy. In: Adam MP, Ardinger $\mathrm{HH}$, Pagon RA, Wallace SE, Bean LH, Stephens K, Amemiya A, editors. GeneReviews ${ }^{\circledR}$ [Internet]. Seattle (WA): University of Washington, Seattle; 1993-2020, updated 2018.

40. Gertler TS, Thompson CH, Vanoye CG et al. Functional consequences of a KCNT1 variant associated with status dystonicus and early-onset infantile encephalopathy. Ann Clin Transl Neurol, 2019. 6(9):1606-1615.

41. Sheffield Diagnostic Genetics Service, www.sheffieldchildrens.nhs.uk/sdgs/next-generationsequencing/, accessed March 2020.

42. Centogene, www.centogene.com/diagnostics/ngs-panels/neurology.html, accessed March 2020.

43. Invitae, www.invitae.com/en/physician/tests/03351/, accessed March 2020.

44. Nagatsu T, Ichinose H. GTP cyclohydrolase I gene, tetrahydrobiopterin, and tyrosine hydroxylase gene: their relations to dystonia and parkinsonism. Neurochem Res, 1996. 21:245-50.

45. Kurian MA, Li Y, Zhen J, Meyer E et al. Clinical and molecular characterisation of hereditary dopamine transporter deficiency syndrome: an observational cohort and experimental study. Lancet Neurol, 2011. 10:54-62.

46. Hewett JW, Zeng J, Niland BP et al. Dystonia-causing mutant torsinA inhibits cell adhesion and neurite extension through interference with cytoskeletal dynamics. Neurobiol Dis, 2006. 22:98-111.

47. Heimer G, Kerätär JM, Riley LG et al. MECR Mutations Cause Childhood-Onset Dystonia and Optic Atrophy, a Mitochondrial Fatty Acid Synthesis Disorder. Am J Hum Genet, 2016. 99:1229-1244.

48. Tieng V, Stoppini L, Villy $S$ et al. Engineering of midbrain organoids containing long-lived dopaminergic neurons. Stem Cells Dev, 2014. 23(13):1535-47. 